\title{
Cytotoxic T Lymphocytes and Vaccine Development
}

\author{
Zhengguo Xiao, ${ }^{1}$ Kim Klonowski, ${ }^{2}$ and Hanchun Yang $^{3}$ \\ ${ }^{1}$ Department of Animal and Avian Sciences, University of Maryland, College Park, MD 20742, USA \\ ${ }^{2}$ Department of Cellular Biology, University of Georgia, Athens, GA 30602, USA \\ ${ }^{3}$ Department of Veterinary Microbiology and Immunology, College of Veterinary Medicine, China Agricultural University, \\ Beijing 100083, China
}

Correspondence should be addressed to Zhengguo Xiao, xiao0028@umd.edu

Received 2 August 2010; Accepted 2 August 2010

Copyright () 2010 Zhengguo Xiao et al. This is an open access article distributed under the Creative Commons Attribution License, which permits unrestricted use, distribution, and reproduction in any medium, provided the original work is properly cited.

Along with the development of new technology, the molecular mechanisms of the generation of functional CTLs have been elucidated over the last several years which together will undoubtedly enhance our progression towards the development of successful vaccines for the many chronic viral infections and cancer. It has been shown that the activation of naïve CTLs requires all three signals to be fully activated: antigen, costimulation, and third signal, which can be provided by inflammatory cytokines such as IL-12 and type I IFNs. In addition, differentiation of memory CTLs requires survival signals and $\gamma_{\mathrm{c}}$-cytokines such as IL2, IL-7, and IL-15. With the booming information on CTLs activation and their application, we felt it was in urgent need to launch a special issue focusing on the most recent progress on CTL research.

We expected an active response to our call for this special issue and indeed are satisfied with the large number of submissions and quick and accurate response of authors to all the reviews and editorial comments. The selected research manuscripts cover a broad area of CTL research, including studies to define new strategies for vaccination against devastating chronic infections like HIV, tumor therapies defining novel tumor-specific epitopes for CTLs, and strategies to enhance cancer control. Interesting attempts have been made to indentify nontraditional adjuvants such as bacteriophages and $\beta$-glucan oligosaccharides while other groups have used unique vectors and cytokines for vaccination studies. Interestingly, this issue attracted a large number of review articles which address the mechanisms of CTL activation, new function of CTLs, vaccine development for viral infections, new adjuvant exploration, and the most upto-date progress in cancer vaccine and cancer therapy.

We thank all of the authors for their enthusiasm in submitting their manuscripts to this special issue and appreciate their efforts and time in process of their manuscripts. Acknowledgement should certainly go to all of the reviewers, most of whom reviewed at least two versions of the same manuscript. Their sacrifice of research time is highly appreciated. We hope the original scientific discoveries and summarized information within this special issue will be helpful and promote new avenues of exploration for scientists studying CTLs in the context of vaccine development.

Zhengguo Xiao Kim Klonowski Hanchun Yang 


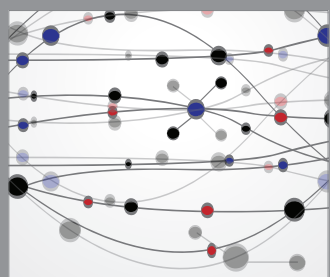

The Scientific World Journal
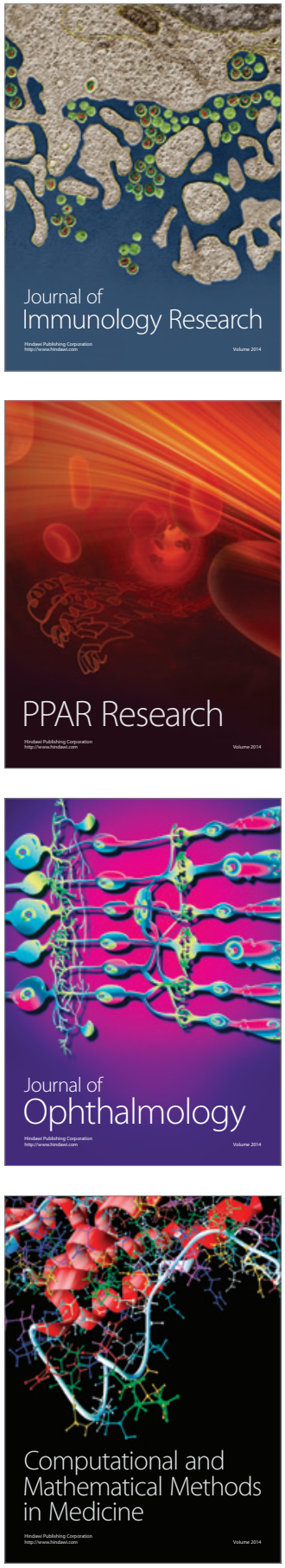

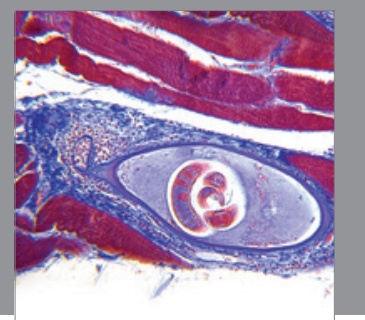

Gastroenterology

Research and Practice
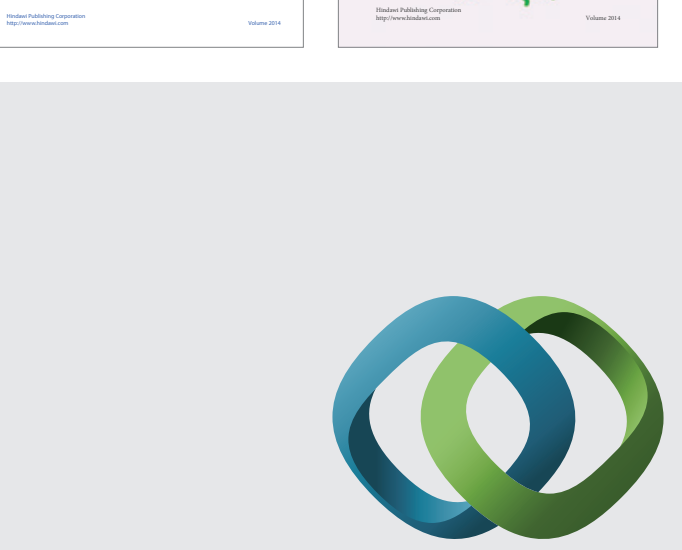

\section{Hindawi}

Submit your manuscripts at

http://www.hindawi.com
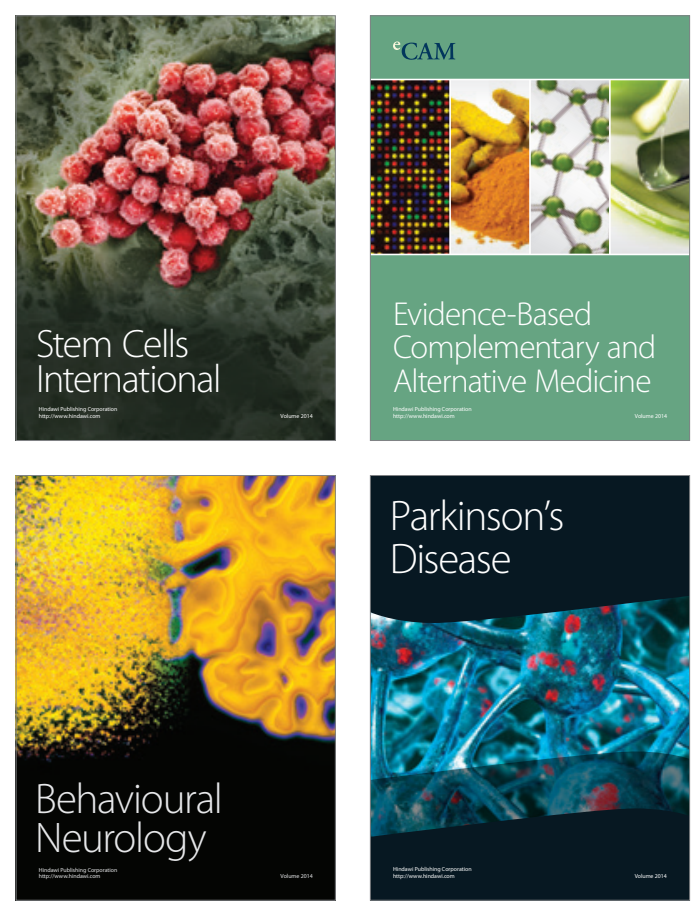

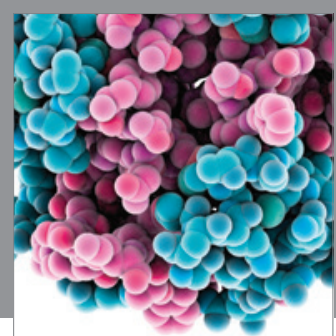

Journal of
Diabetes Research

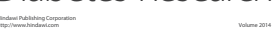

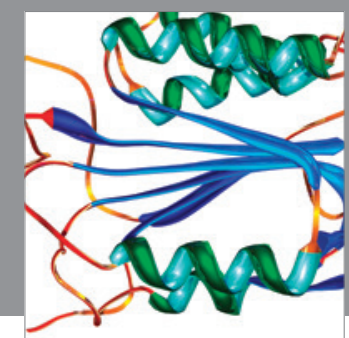

Disease Markers
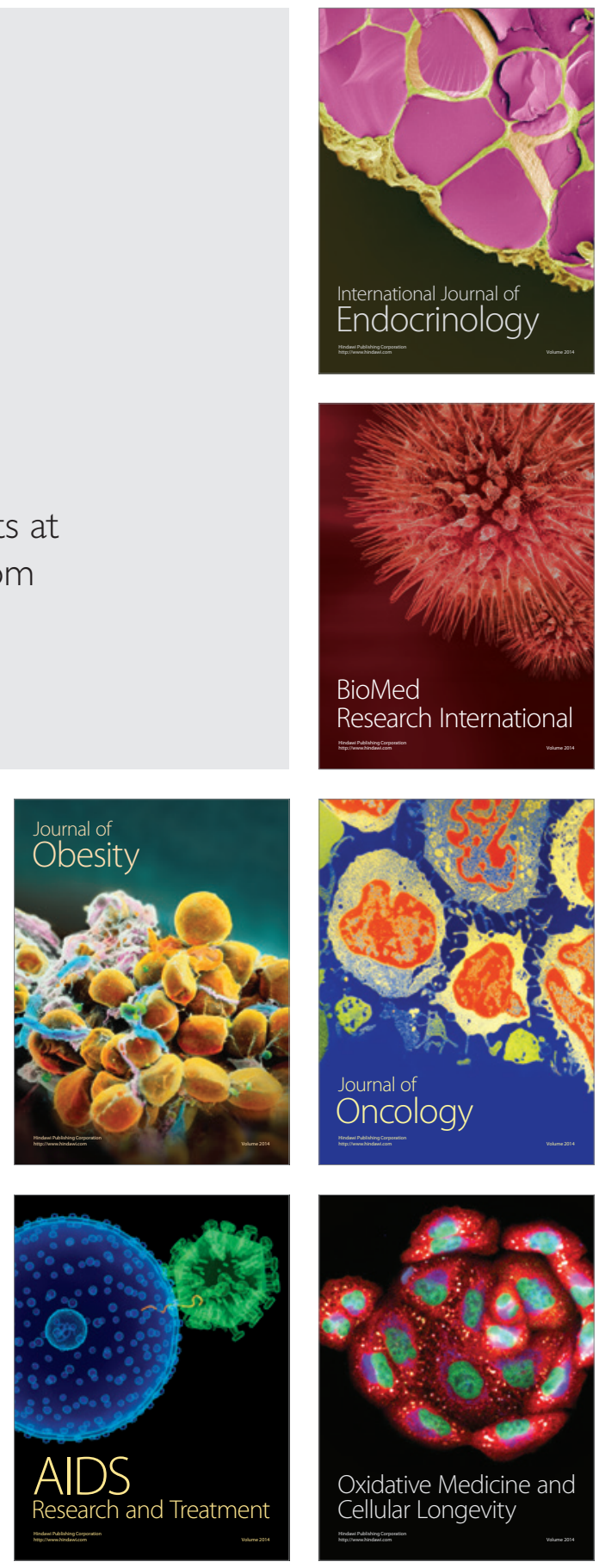